\title{
Surface tension of hot and dense quark matter under strong magnetic fields
}

\author{
G. Lugones ${ }^{1}$ and A. G. Grunfeld ${ }^{2,3}$ \\ ${ }^{1}$ Centro de Ciências Naturais e Humanas, Universidade Federal do ABC, Avenida dos Estados 5001, CEP 09210-580, Santo André, \\ São Paulo, Brazil \\ ${ }^{2}$ CONICET, Rivadavia 1917, (1033) Buenos Aires, Argentina \\ ${ }^{3}$ Departamento de Física, Comisión Nacional de Energía Atómica, Avenida Libertador 8250, (1429) Buenos Aires, Argentina
}

(Received 25 November 2018; published 29 March 2019)

\begin{abstract}
We study the surface tension of hot, highly magnetized three-flavor quark matter droplets, focusing specifically on the thermodynamic conditions prevailing in neutron stars, hot lepton-rich protoneutron stars, and neutron star mergers. We explore the role of temperature, baryon number density, trapped neutrinos, droplet size, and magnetic fields within the multiple reflection expansion formalism (MRE), assuming that astrophysical quark matter can be described as a mixture of free Fermi gases composed of quarks $u, d$, s, electrons, and neutrinos, in chemical equilibrium under weak interactions. We find that the total surface tension is rather unaffected by the size of the drop but is quite sensitive to the effect of baryon number density, temperature, trapped neutrinos, and magnetic fields (especially above $e B \sim 5 \times 10^{-3} \mathrm{GeV}^{2}$ ). Surface tensions parallel and transverse to the magnetic field span values up to $\approx 25 \mathrm{MeV} / \mathrm{fm}^{2}$. For $T \lesssim 100 \mathrm{MeV}$, the surface tension is a decreasing function of temperature but above $100 \mathrm{MeV}$ it increases monotonically with $T$. Finally, we discuss some astrophysical consequences of our results.
\end{abstract}

DOI: 10.1103/PhysRevC.99.035804

\section{INTRODUCTION}

The study of the surface tension of quark matter has attracted much attention recently because of its impact on heavy-ion collisions and in astrophysics. In the astrophysical context, quark matter surface tension plays a key role in the understanding of neutron star (NS) interiors (see Refs. [1,2] and references therein). In fact, it has been speculated that hybrid stars may contain a mixed hadron-quark phase in their interiors. In such a phase, it is assumed that the electric charge is zero globally but not locally, and therefore charged hadronic and quark matter may share a common lepton background, leading to a quark-hadron mixture extending over a wide density region of the star [3]. The mixed phase entails a smooth variation of the energy density, leading in turn to a continuous density profile along the star. Whether the quark-hadron interface in a compact star is actually a sharp discontinuity or a wide mixed region depends crucially on the amount of surface energy needed for the formation of drops of one phase embedded in the other [4,5]. If the energy cost of surface effects exceeds the gain in bulk energy, the scenario involving a sharp interface turns out to be favorable. The quark matter surface tension is also crucial for understanding the most external layers of strange quark stars [6], where matter may fragment into a charge-separated mixture, involving positively charged strangelets immersed in a negatively charged sea of electrons, presumably forming a crystalline solid crust [7]. This scenario would be favored below some critical surface tension, which is typically of the order of a few $\mathrm{MeV} / \mathrm{fm}^{2}$ [8]. However, in spite of its relevance for NS physics, the surface tension is still poorly known for quark matter. Early calculations as well as recent calculations within different models give rather low values [1,9-12], typically below $\approx 30 \mathrm{MeV} / \mathrm{fm}^{2}$, but significantly larger results (above $\approx 100 \mathrm{MeV} / \mathrm{fm}^{2}$ ) were obtained by other authors [2,13-15].

In this work, we study the surface tension of hot, highly magnetized three-flavor quark matter droplets within the multiple reflection expansion (MRE) framework (for details on the MRE formalism, see Refs. [16-19] and references therein). We describe quark matter as a strongly magnetized free Fermi gas of $u, d$, and $s$ quarks, electrons, and neutrinos, all in chemical equilibrium under weak interactions [1,20]. We focus on the thermodynamic conditions prevailing in NSs, hot lepton-rich protoneutron stars (PNSs), and binary NS mergers. NSs are believed to be born after the gravitational collapse and supernova explosion of a massive star. Initially, PNSs are very hot and lepton-rich objects, where neutrinos are temporarily trapped. Initially, the temperature may reach $40 \mathrm{MeV}$ and the chemical potential of trapped neutrinos can be as high as $150 \mathrm{MeV}$. During the first minute of evolution, the PNS evolves to form a cold NS. As neutrinos are radiated, the lepton-per-baryon content of matter goes down and the neutrino chemical potential tends to essentially zero. Finally, binary NS merging has recently gained attention after the LIGO/Virgo Collaboration detected the gravitational waves from the merging event GW170817 [21]. Numerical simulations of these events suggest that the just-merged compact object may attain high temperatures [22,23] (as high as $\approx 70 \mathrm{MeV}$ [24]) with an expected huge amount of trapped neutrinos. As a limiting scenario, we will consider here an extreme configuration with $T=100 \mathrm{MeV}$ and $\mu_{v_{e}}=200 \mathrm{MeV}$.

The article is organized as follows: In Sec. II, we present the MRE formalism for finite-size droplets immersed in a strong magnetic field. In Sec. III, we include finite-size effects in the thermodynamic quantities. In particular, we derive the 
longitudinal and transverse surface tension as well as the particle number density. We also introduce some thermodynamic constraints and present different astrophysical scenarios to be analyzed. In Sec. IV, we report and discuss our results. Finally, in Sec. V, we present our conclusions.

\section{FINITE-SIZE EFFECTS IN THE PRESENCE OF A MAGNETIC FIELD}

In the MRE formalism, the modified density of states of a finite droplet with an arbitrary shape is given by [16,17]

$$
\rho_{\mathrm{MRE}}(k, m, S, V, \ldots)=1+\frac{2 \pi^{2}}{k} \frac{S}{V} f_{S}+\cdots,
$$

where $m$ is the mass of a quark or a lepton, $k$ is its momentum, $S$ is the droplet's surface, $V$ is its volume, and

$$
f_{S}(k)=-\frac{1}{8 \pi}\left(1-\frac{2}{\pi} \arctan \frac{k}{m}\right)
$$

is the surface contribution to the new density of states. As pointed out in Refs. $[18,19]$ the MRE density of states given in Eq. (1) may become negative for small momenta. To exclude this nonphysical behavior, an infrared cutoff $\Lambda$ is introduced in momentum space (see Ref. [19] for details), where $\Lambda$ is the largest solution of the equation $\rho_{\mathrm{MRE}}(k, m, S, V, \ldots)=0$ with respect to the momentum $k$.

To implement the MRE formalism, we have to perform the following replacement in a generic thermodynamic integral $I$ :

$$
I \equiv \frac{1}{(2 \pi)^{3}} \int \cdots d^{3} k \rightarrow \frac{1}{(2 \pi)^{3}} \int_{\Lambda}^{\infty} \cdots \rho_{\mathrm{MRE}}(k) 4 \pi k^{2} d k
$$

For matter immersed in a magnetic field $\mathbf{B}$ pointing in the $z$ direction, the transverse motion of particles with electric charge qe is quantized into Landau levels (LL). The momentum $k$ in Eqs. (1) and (2) is given by $k=\sqrt{k_{z}^{2}+2 v|q e B|}$, where $v \geqslant 0$ is an integer, and the momentum integrals in the transverse plane must be replaced by sums over the discretized levels. Thus, in the thermodynamic integrals, we must use [1]

$$
I \rightarrow \frac{|q e B|}{2 \pi^{2}} \sum_{\nu=0}^{\infty} \alpha_{\nu} \int_{\Lambda_{v}}^{\infty} \cdots \rho_{\mathrm{MRE}} d k_{z}
$$

where $\Lambda_{v}$ are the cutoffs in the momentum along the direction of the magnetic field, which now depend on the Landau levels. $\alpha_{v}=2$ for all cases except for $v=0$, where $\alpha_{v}=1$.

The infrared cutoff $\Lambda_{v}$ is the largest solution of the equation $\rho_{\mathrm{MRE}}\left(k_{z}, m, S, V\right)=0$ with respect to the momentum $k_{z}$. Taking only the first two terms of Eq. (1), we obtain [1]

$$
\Lambda_{\nu}=\sqrt{\frac{S^{2}}{4 V^{2}} x_{0}^{2}-2 v|q e B|},
$$

where $x_{0}$ is the solution of $\lambda x=\cot x$ with $\lambda=S /(2 V m)$. In Table I, we show the values of $x_{0}$ for different quark masses and different values of $V / S$.
TABLE I. Values of the parameter $x_{0}$ for different particle masses and different values of $V / S$.

\begin{tabular}{lccc}
\hline \hline Particles & $m[\mathrm{MeV}]$ & $V / S[\mathrm{fm}]$ & $x_{0 i}$ \\
\hline Electrons & 0.511 & 10 & 0.225631 \\
& 0.511 & 50 & 0.487927 \\
& 0.511 & 100 & 0.663088 \\
Quarks $u, d$ & 0.511 & $\infty$ & $\pi / 2$ \\
& 5 & 10 & 0.657008 \\
& 5 & 50 & 1.14604 \\
Quarks $s$ & 5 & 100 & 1.31661 \\
& 5 & $\infty$ & $\pi / 2$ \\
& 150 & 10 & 1.47413 \\
& 150 & 50 & 1.5504 \\
& 150 & 100 & 1.56053 \\
\hline \hline
\end{tabular}

The thermodynamic integrals can be written in terms of the particle's energy $E=\left(k_{z}^{2}+2 \nu|q e B|+m^{2}\right)^{1 / 2}$ as

$$
I \rightarrow \frac{|q e B|}{2 \pi^{2}} \sum_{\nu=0}^{\infty} \alpha_{v} \int_{\ell}^{\infty} \cdots \rho_{\mathrm{MRE}}(E) \frac{E d E}{k_{z}(E)}
$$

where $\quad k(E)=\left(E^{2}-m^{2}\right)^{1 / 2}, \quad k_{z}(E)=\left(E^{2}-2 \nu|q e B|-\right.$ $\left.m^{2}\right)^{1 / 2}$, and the energy cutoff $\ell=\sqrt{\Lambda_{v}^{2}+2 \nu|q e B|+m^{2}}$ reads

$$
\ell=\sqrt{\frac{S^{2}}{4 V^{2}} x_{0}^{2}+m^{2}}
$$

Notice that $\ell$ does not depend on the index $\nu$.

To conclude, it is worth mentioning that the surface tension of strange quark matter has also been studied recently within the MRE model [25]. In fact, in that reference the authors compare two different approaches for finite-size systems. First, they use the so-called "equivparticle" model to obtain the energy per baryon of strangelets and use a liquid-drop-type formula [26] to fit the obtained results in order to identify the surface tension and the curvature energy. Then, they compare the results obtained by this method with the ones obtained within the MRE. They find that some damping factors must be included in the MRE density of states in order to make these two approaches coincide. In particular, an overall factor is introduced in $f_{S}$ which significantly reduces the value of the surface tension. In the present paper, we focus on the original version of the MRE model.

\section{THERMODYNAMIC QUANTITIES AT FINITE TEMPERATURE}

\section{A. Number density}

The number densities can be obtained starting from [20]

$$
n_{i}(T)=\frac{\left|q_{i} e B\right|}{2 \pi^{2}} \sum_{\nu=0}^{\infty} \alpha_{\nu} \int_{0}^{\infty}\left(F_{i}-\bar{F}_{i}\right) d k_{z}
$$


where the Fermi-Dirac distribution functions for particles and antiparticles are respectively

$$
\begin{aligned}
F_{i} & =\frac{1}{e^{\left(E_{i}-\mu_{i}\right) / T}+1}, \\
\bar{F}_{i} & =\frac{1}{e^{\left(E_{i}+\mu_{i}\right) / T}+1},
\end{aligned}
$$

with $\mu_{i}$ being the chemical potential for the particle species $i$. Including the MRE density of states, we obtain

$$
\begin{aligned}
n_{i}= & \frac{\left|q_{i} e B\right|}{2 \pi^{2}} \sum_{\nu=0}^{\infty} \alpha_{\nu} \int_{\Lambda_{i, v}}^{\infty}\left(F_{i}-\bar{F}_{i}\right)\left[1+\frac{2 \pi^{2} S}{k V} f_{S, i}\right] d k_{z} \\
= & \frac{\left|q_{i} e B\right|}{2 \pi^{2}} \sum_{\nu=0}^{\infty} \alpha_{\nu} \int_{\ell_{i}}^{\infty}\left(F_{i}-\bar{F}_{i}\right) \\
& \times\left[1+\frac{2 \pi^{2} S}{V} \frac{f_{S, i}(E)}{k(E)}\right] \frac{E d E}{k_{z}(E)},
\end{aligned}
$$

where the energy cutoff is $\ell_{i}=\sqrt{S^{2} x_{0 i}^{2} /\left(4 V^{2}\right)+m_{i}^{2}}$.

\section{B. Parallel pressure and surface tension}

A similar procedure allows writing the parallel thermodynamic potential of a magnetized quark matter drop within the MRE formalism as (see Refs. [1,20]):

$$
\begin{aligned}
-\Omega_{i}^{\|}= & \frac{\left|q_{i} e B\right|}{2 \pi^{2}} \sum_{\nu=0}^{\infty} \alpha_{\nu} \int_{\Lambda_{i, v}}^{\infty} \frac{\left(F_{i}+\bar{F}_{i}\right) k_{z}^{2}}{\sqrt{k^{2}+m_{i}^{2}}} \\
& \times\left(V+\frac{2 \pi^{2} f_{S, i}(k)}{k} \times S\right) d k_{z} .
\end{aligned}
$$

The latter expression can be written in the form

$$
\Omega_{i}^{\|}=-\Pi_{i}^{\|} V+\alpha_{i}^{\|} S,
$$

where $\Pi_{i}^{\|}$is the parallel pressure within the MRE formalism and $\alpha_{i}^{\|}$is the parallel surface tension, i.e.,

$$
\begin{aligned}
\Pi_{i}^{\|}(T) & =\frac{\left|q_{i} e B\right|}{2 \pi^{2}} \sum_{\nu=0}^{\infty} \alpha_{\nu} \int_{\Lambda_{i, v}}^{\infty} \frac{\left(F_{i}+\bar{F}_{i}\right) k_{z}^{2} d k_{z}}{\sqrt{k^{2}+m_{i}^{2}}} \\
& =\frac{\left|q_{i} e B\right|}{2 \pi^{2}} \sum_{\nu=0}^{\infty} \alpha_{\nu} \int_{\ell_{i}}^{\infty}\left(F_{i}+\bar{F}_{i}\right) k_{z}(E) d E, \\
\alpha_{i}^{\|}(T) & =-\left|q_{i} e B\right| \sum_{\nu=0}^{\infty} \alpha_{\nu} \int_{\Lambda_{i, v}}^{\infty} \frac{\left(F_{i}+\bar{F}_{i}\right) f_{S, i} k_{z}^{2} d k_{z}}{k \sqrt{k^{2}+m_{i}^{2}}} \\
& =-\left|q_{i} e B\right| \sum_{\nu=0}^{\infty} \alpha_{\nu} \int_{\ell_{i}}^{\infty}\left(F_{i}+\bar{F}_{i}\right) \frac{f_{S, i} k_{z}(E) d E}{k(E)} .
\end{aligned}
$$

To obtain the total parallel surface tension, we have to add the contribution of all particle species, i.e., quarks $u, d, s$ and electrons:

$$
\alpha_{\mathrm{tot}}^{\|}=\sum_{i=u, d, s, e} \alpha_{i}^{\|}
$$

Within the MRE formalism, neutrinos have vanishing surface tension because they are assumed to be massless; then from Eq. (2), $f_{S, v_{e}}=0$.

\section{Transverse pressure and surface tension}

The transverse thermodynamic potential of a magnetized quark matter drop reads $[1,20]$

$$
-\Omega_{i}^{\perp}=\frac{V\left|q_{i} e B\right|^{2}}{2 \pi^{2}} \sum_{\nu=0}^{\infty} \alpha_{\nu} v \int_{\Lambda_{i, v}}^{\infty} \frac{\left(F_{i}+\bar{F}_{i}\right) \rho_{\mathrm{MRE}, i} d k_{z}}{\sqrt{k^{2}+m_{i}^{2}}} .
$$

Again, the latter expression has the form

$$
\Omega_{i}^{\perp}=-\Pi_{i}^{\perp} V+\alpha_{i}^{\perp} S .
$$

Therefore, the transverse pressure $\Pi_{i}^{\perp}$ and the transverse surface tension $\alpha_{i}^{\perp}$ are given by

$$
\begin{aligned}
\Pi_{i}^{\perp}(T) & =\frac{\left|q_{i} e B\right|^{2}}{2 \pi^{2}} \sum_{\nu=0}^{\infty} \alpha_{\nu} v \int_{\Lambda_{i, v}}^{\infty} \frac{\left(F_{i}+\bar{F}_{i}\right) d k_{z}}{\sqrt{k^{2}+m_{i}^{2}}} \\
& =\frac{\left|q_{i} e B\right|^{2}}{2 \pi^{2}} \sum_{\nu=0}^{\infty} \alpha_{\nu} v \int_{\ell_{i}}^{\infty} \frac{\left(F_{i}+\bar{F}_{i}\right)}{k_{z}(E)} d E, \\
\alpha_{i}^{\perp}(T) & =-\left|q_{i} e B\right|^{2} \sum_{\nu=0}^{\infty} \alpha_{\nu} v \int_{\Lambda_{i, v}}^{\infty} \frac{\left(F_{i}+\bar{F}_{i}\right) f_{S, i} d k_{z}}{k \sqrt{k^{2}+m_{i}^{2}}} \\
& =-\left|q_{i} e B\right|^{2} \sum_{\nu=0}^{\infty} \alpha_{\nu} v \int_{\ell_{i}}^{\infty} \frac{\left(F_{i}+\bar{F}_{i}\right) f_{S, i} d E}{k(E) k_{z}(E)},
\end{aligned}
$$

where $i$ runs over all particle species, e.g., quarks $u, d, s$ and electrons. The total transverse surface tension is

$$
\alpha_{\mathrm{tot}}^{\perp}=\sum_{i=u, d, s, e} \alpha_{i}^{\perp} .
$$

Here we also have $\alpha_{v_{e}}^{\perp}=0$.

\section{Thermodynamic constrains and astrophysical scenarios}

In this work, we are interested in potential astrophysical applications; therefore, we shall consider that quark matter is electrically neutral and in equilibrium under weak interactions. Chemical equilibrium is maintained by weak interactions among quarks, e.g., $d \leftrightarrow u+e^{-}+\bar{v}_{e}, s \leftrightarrow u+e^{-}+\bar{v}_{e}$, $u+d \leftrightarrow u+s$, from which we obtain the following relations between the chemical potentials:

$$
\begin{aligned}
\mu_{d} & =\mu_{u}+\mu_{e}-\mu_{v_{e}}, \\
\mu_{s} & =\mu_{d} .
\end{aligned}
$$

Local charge neutrality means that

$$
\frac{2}{3} n_{u}-\frac{1}{3} n_{d}-\frac{1}{3} n_{s}-n_{e}=0,
$$

where the number densities are given by Eq. (11).

We focus here in thermodynamic conditions that are relevant for three different astrophysical scenarios:

(1) Cold deleptonized NSs. This is the case of most NSs a few minutes after their birth. The thermodynamic state can be characterized by a very low temperature (typically below $1 \mathrm{MeV}$ ) and no trapped neutrinos because their mean free path is much larger that the stellar 

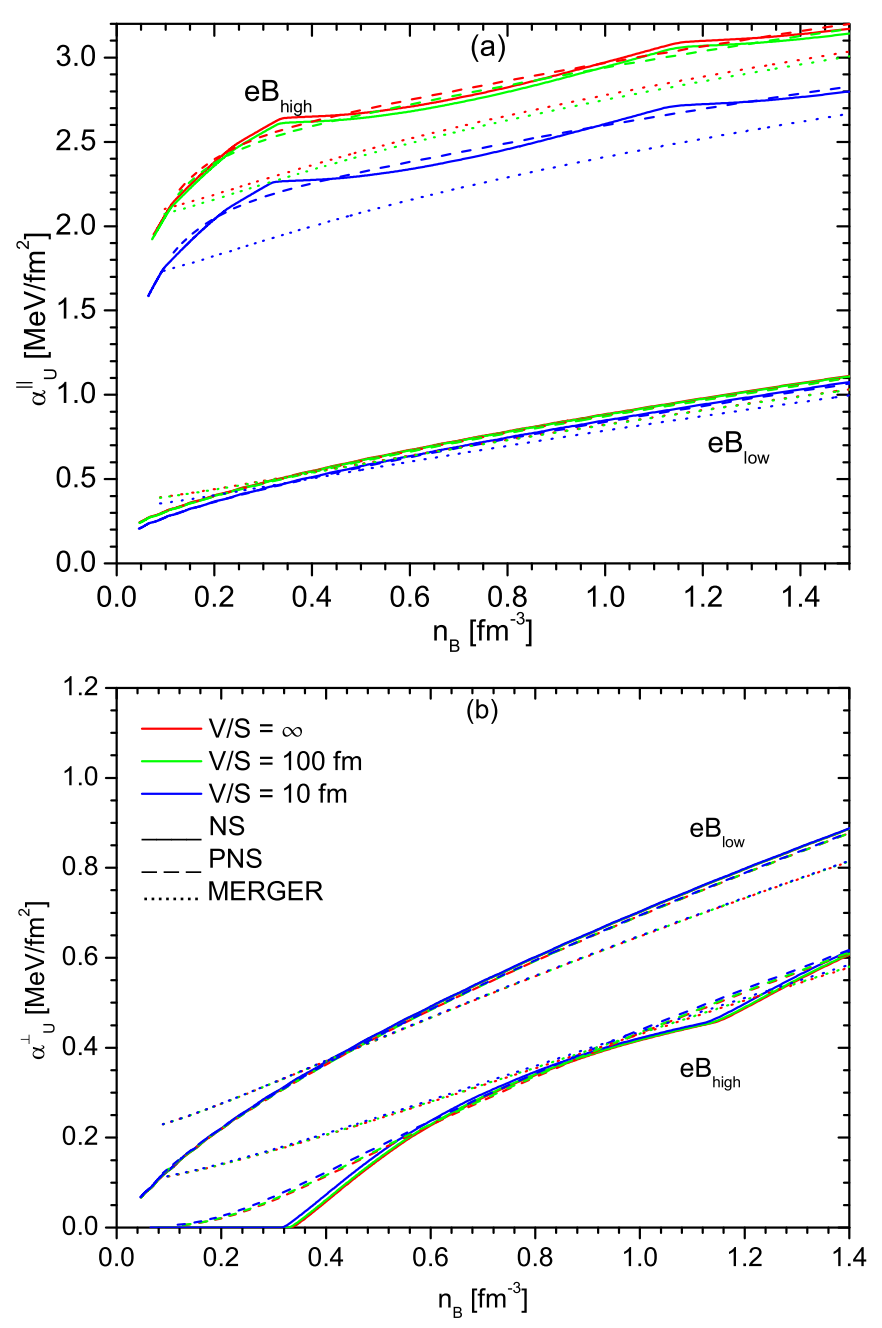

FIG. 1. Surface tension for quarks $u$ in the parallel direction [panel (a)] and in the transverse direction [panel (b)]. The results are shown for drops with $V / S=10 \mathrm{fm}, 100 \mathrm{fm}$, and for the bulk limit with $V / S=\infty$. The magnetic field intensities are $e B_{\text {low }}=5 \times$ $10^{-3} \mathrm{GeV}^{2}$ and $e B_{\text {high }}=5 \times 10^{-2} \mathrm{GeV}^{2}$. We considered the three different astrophysical scenarios described in the text.

radius. As a representative case, we shall consider here $T=1 \mathrm{MeV}$ and $\mu_{\nu_{e}}=0$. This is essentially the same case already studied in our previous work [1] but we include it here for comparison.

(2) Hot lepton-rich PNSs. This is the case of NSs during the first few minutes after their birth in corecollapse supernovae. The thermodynamic state can be characterized by high temperatures (typically up to $\approx 40 \mathrm{MeV}$ ) and a large amount of trapped neutrinos in the system (neutrino chemical potential $\mu_{v_{e}}$ up to $\approx 150 \mathrm{MeV}$ ) [27]. As a representative case, we consider here $T=30 \mathrm{MeV}$ and $\mu_{v_{e}}=100 \mathrm{MeV}$.

(3) Binary NS mergers (labeled as MERGER in the figures). Very recently, the LIGO/Virgo Collaboration detected the first signal of gravitational waves coming from the binary NS merger GW170817 [21]. Numerical simulations of these events suggest that the just merged compact object may attain very high
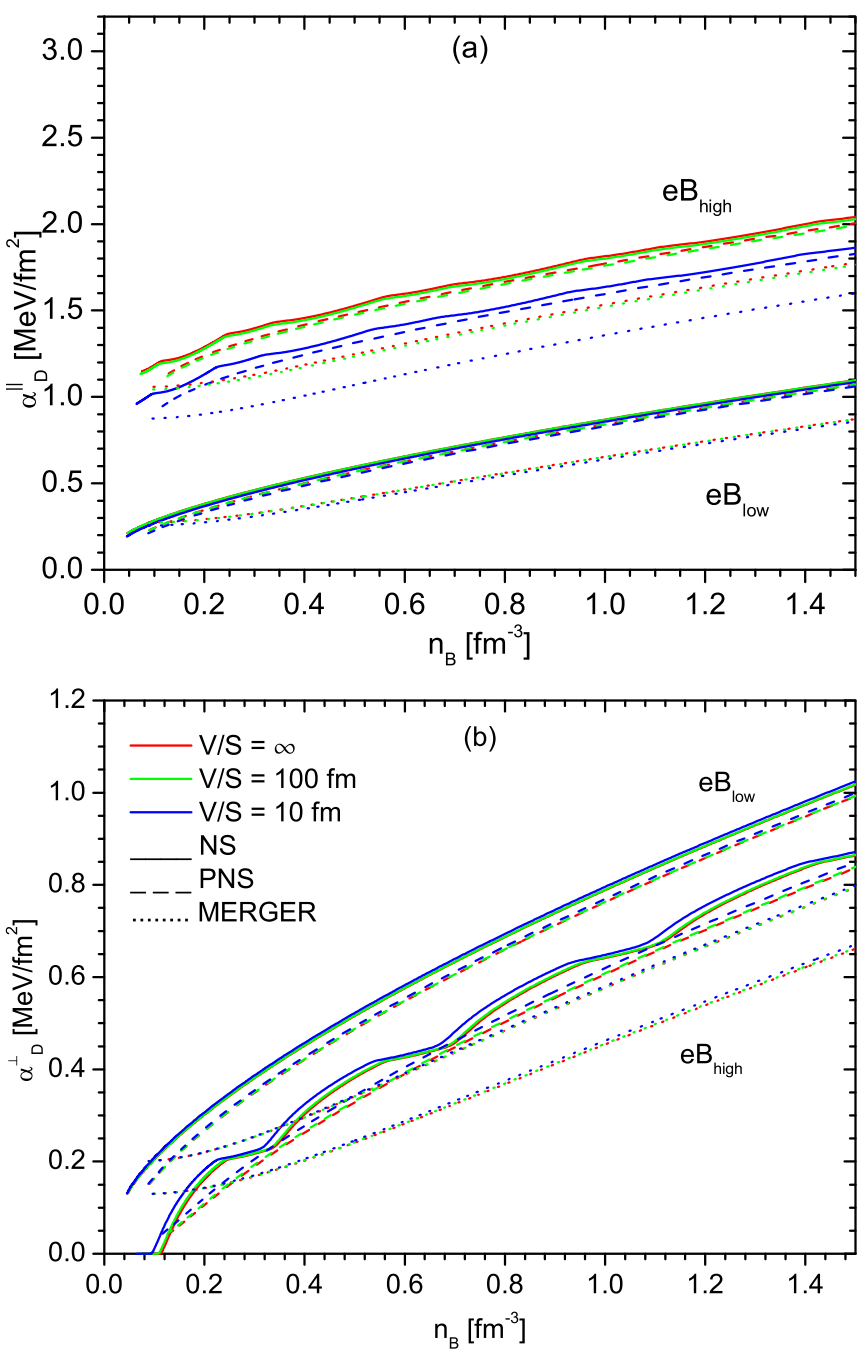

FIG. 2. Same as in Fig. 1 but for quarks $d$.

temperatures (up to $70 \mathrm{MeV}$ [24]) and a huge amount of trapped neutrinos can be expected. As a limiting case, we consider here $T=100 \mathrm{MeV}$ and $\mu_{v_{e}}=$ $200 \mathrm{MeV}$.

\section{RESULTS}

In this section, we show our results for the longitudinal $\left(\alpha_{f}^{\|}\right)$ and transverse surface tension $\left(\alpha_{f}^{\perp}\right)$, focusing on their dependence on the the baryon number density $n_{B}=\frac{1}{3}\left(n_{u}+n_{d}+\right.$ $n_{s}$ ), the temperature, the magnetic field strength $e B$, the drop's size $V / S$, and the neutrino chemical potential. As emphasized in our previous work [1], $\alpha_{f}^{\|}$and $\alpha_{f}^{\perp}$ do not depend on the exact geometry of the drop but only on the ratio $V / S$, which is taken here as a free parameter (we adopt $V / S=10 \mathrm{fm}$, $100 \mathrm{fm}$, and the bulk limit with $\mathrm{V} / \mathrm{S}=\infty$ ). For the magnetic field strength, we consider a low value and a high one: $e B_{\text {low }}=$ $5 \times 10^{-3} \mathrm{GeV}^{2}$ and $e B_{\text {high }}=5 \times 10^{-2} \mathrm{GeV}^{2}$. For magnetic fields smaller than $e B_{\text {low }}$, the results are the same as for $e B_{\text {low }}$, as already emphasized in our previous work [1].

In Figs. 1-4, we show $\alpha_{f}^{\|}$and $\alpha_{f}^{\perp}$, for $u, d, s$ quarks and electrons as a function of the baryon number density. For 

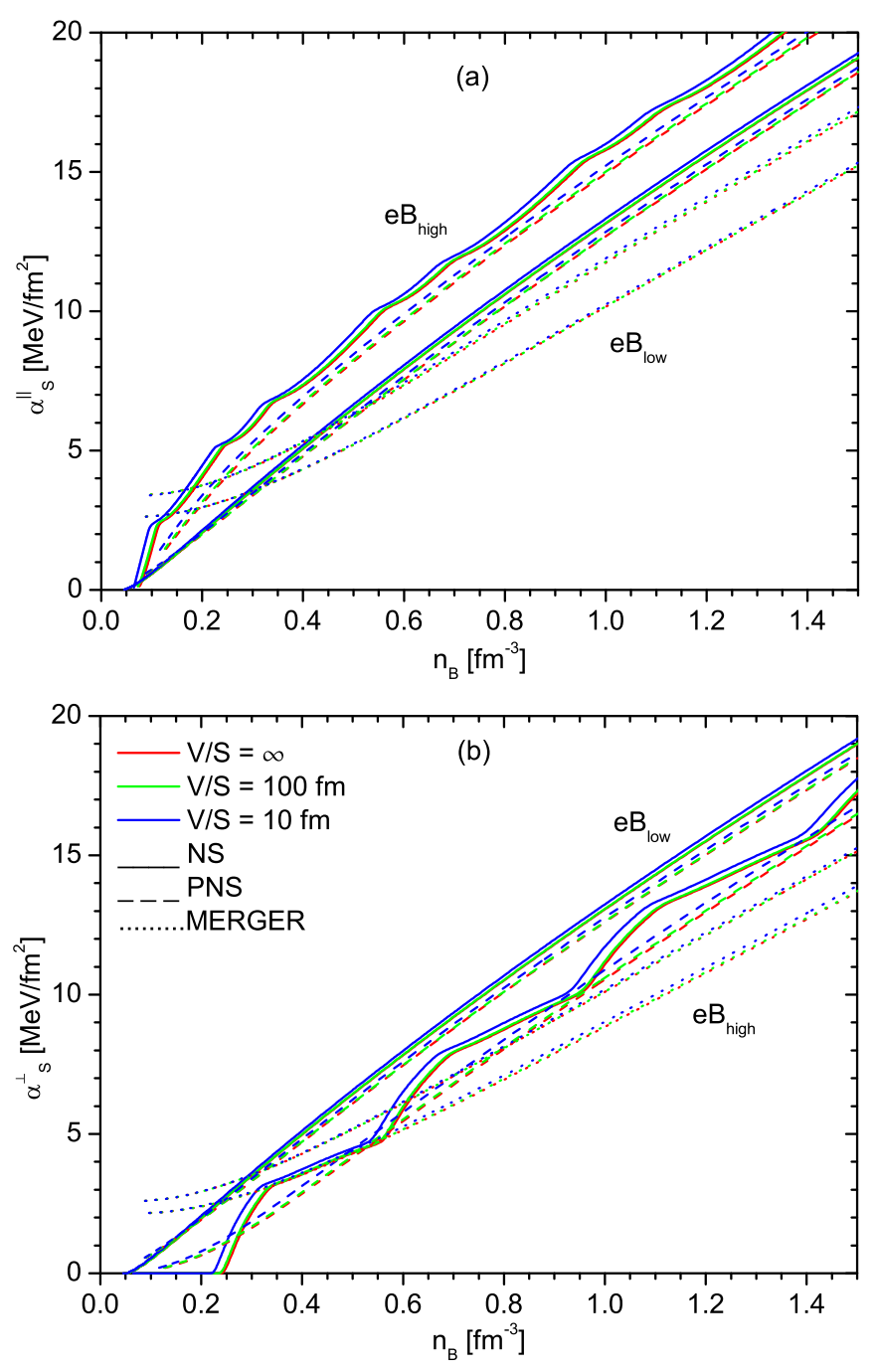

FIG. 3. Same as in Fig. 1 but for quarks $s$.

electrons, we show only the longitudinal contribution because the perpendicular contribution is negligible.

As shown Figs. 1-3, the surface tension of $s$ quarks is significantly larger than for nonstrange quarks ( $u$ or $d$ ). This is essentially a mass effect, because the surface contribution $f_{S}$ to the density of states is larger for the more massive particles. However, when comparing quark species with the same mass such as $u$ and $d$ quarks, the role of other properties becomes more apparent. For example, the difference of a factor of $\approx 2$ between $\alpha_{u}^{\|}$and $\alpha_{d}^{\|}$[see Figs. 1(a) and 2(a)] is mainly due to the overall factor $q B$ in the parallel component $\left(\left|q_{u} / q_{d}\right|=\right.$ 2). An explanation of the difference between $\alpha_{u}^{\perp}$ and $\alpha_{d}^{\perp}$ is more tricky. There is an overall factor of 4 between them $\left(\left|q_{u} / q_{d}\right|^{2}=4\right)$ and the infrared cutoff is smaller for $d$ quarks than for $u$ quarks. However, the effect that leads to more significant differences between $\alpha_{u}^{\perp}$ and $\alpha_{d}^{\perp}$ is related to the chemical potential of $u$ and $d$ species. In fact, since the lowest Landau level does not contribute to the transverse component, the result depends on the filling of the excited Landau levels, which tend to be more populated for the particle species with larger chemical potential. For quark matter in chemical

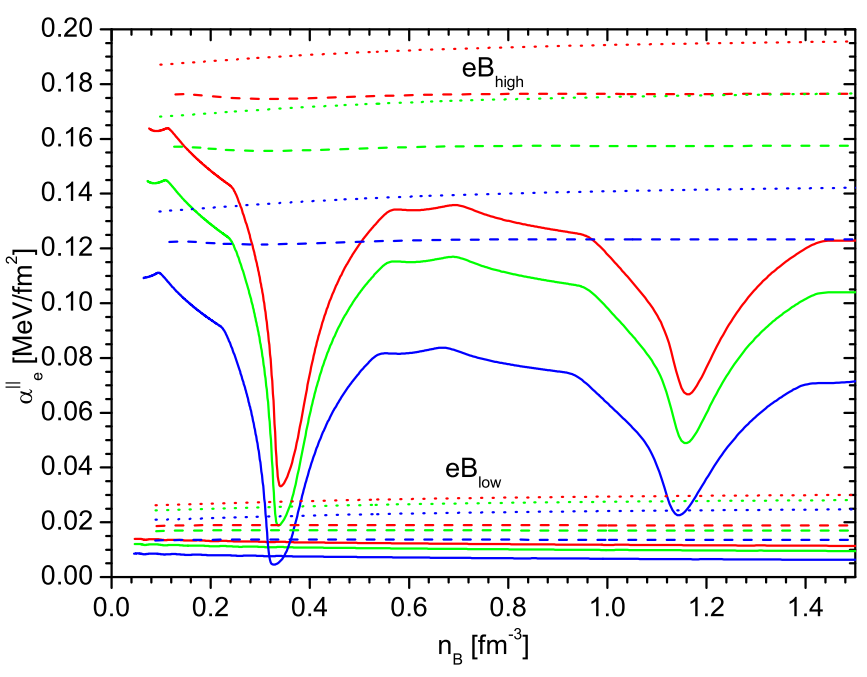

FIG. 4. Parallel surface tension for electrons. The transverse surface tension is not shown because it is negligible. Lines follow the same pattern as in previous figures.

equilibrium under weak interactions, we have in general that $\mu_{d}>\mu_{u}$. This explains why the transverse component is larger for $d$ quarks than for $u$ quarks [see Figs. 1(b) and 2(b)].

As apparent from Figs. 1-3, for $u, d$, and $s$ quarks, the surface tension is an increasing function of the baryon number density, for fixed values of $e B, V / S, T$, and $\mu_{v_{e}}$. In all cases, $\alpha_{f}^{\|}$is larger for $e B_{\text {high }}$ than for $e B_{\text {low }}$. For the transverse component, the behavior is the opposite, i.e., $\alpha_{f}^{\perp}$ decreases as the magnetic field increases. For very high values of the magnetic field $e B_{\text {high }}$, the curves for the NS case clearly show de Haas-van Alphen oscillations related to the filling of new Landau levels. For lower fields, such oscillations are also apparent for the lightest particles. However, for the PNS and MERGER cases, the high temperature allows several excited Landau levels to be fulfilled at any value of the magnetic field. As a consequence, de Haas-van Alphen oscillations are smoothed out.

The effect of varying $V / S$ is minor for heavier particles, like strange quarks (see Fig. 3). For $u$ quarks, the parallel surface tension for $V / S=10 \mathrm{fm}$ is decreased with respect to the bulk case by $\approx 10 \%$ for $e B_{\text {low }}$ and by $\approx 20 \%$ for $e B_{\text {high }}$. For $d$ quarks, the effect is qualitatively the same but quantitatively smaller. The transverse surface tension is almost unaffected by $V / S$ for any value of $e B$. In the case of electrons, the effect of $V / S$ is even larger, because they are lighter.

Now let us focus on the behavior of the surface tension in different astrophysical scenarios. In the PNS and MERGER cases, the results are affected by neutrino trapping in spite of the vanishing surface tension of the neutrino gas. The influence of neutrinos enters only through the chemical equilibrium condition. In the PNS and MERGER cases, the curves are smoother than in the NS case. This is because at finite temperatures the Fermi surface has a thickness of order $T$ which allows Landau levels to be fulfilled more gradually than in the NS case. The results for the NS case are in agreement with the results found in our previous work [1]. For all flavors, 

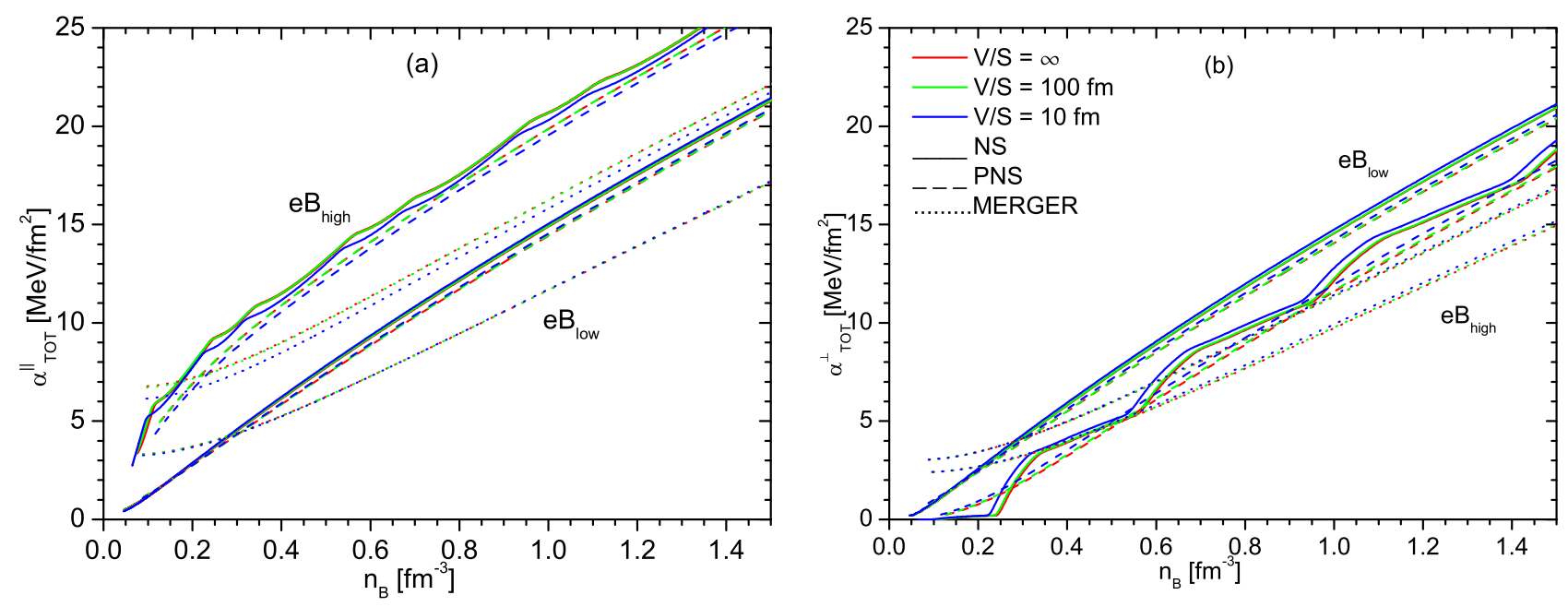

FIG. 5. Total surface tension obtained by summing the contributions of quarks $u, d, s$ and electrons in the parallel direction [panel (a)] and in the transverse direction [panel (b)].
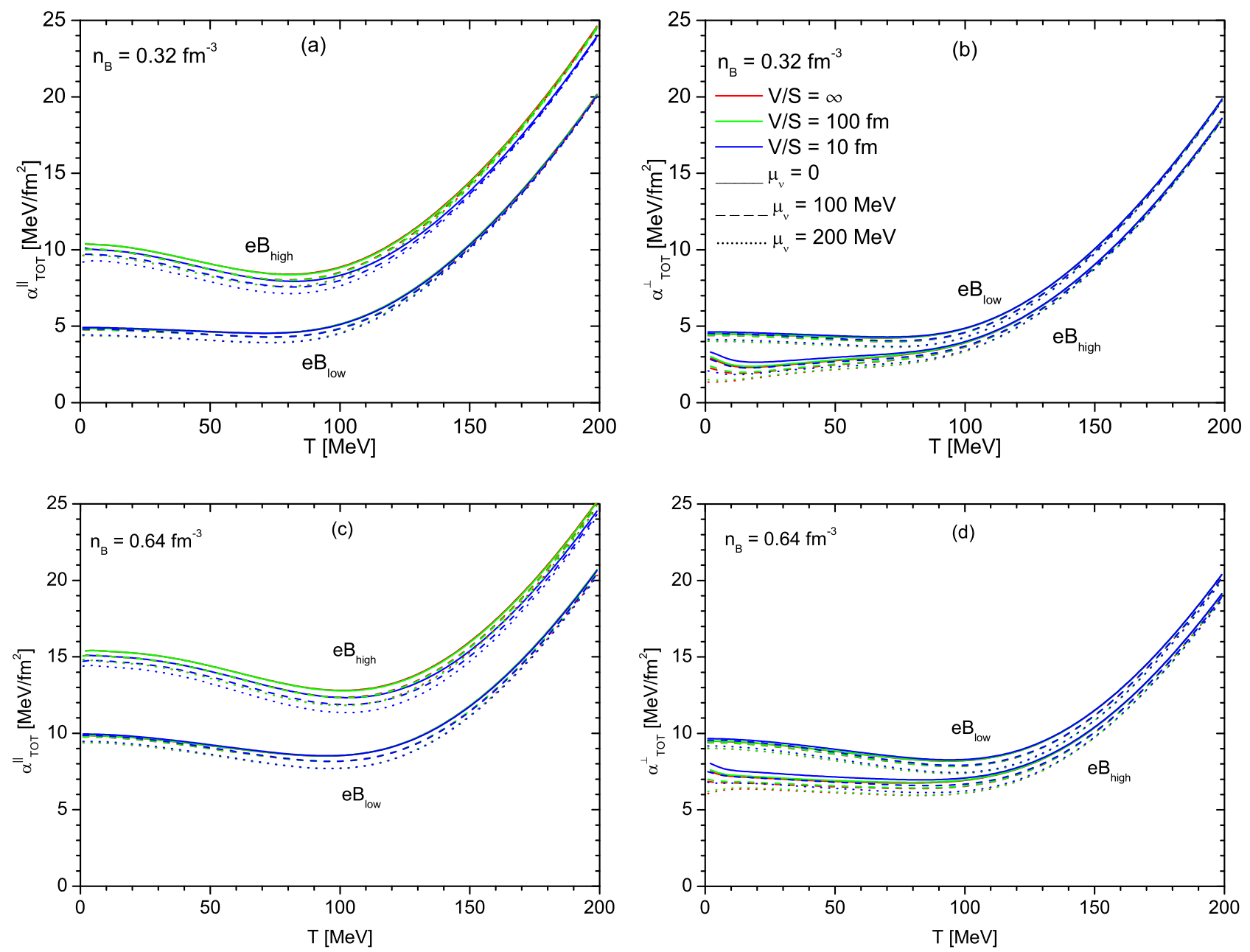

FIG. 6. Total surface tension obtained by summing the contributions of quarks $u, d, s$ and electrons in the parallel direction [panels (a) and (c)] and in the transverse direction [panels (b) and (d)], as a function of the temperature, for fixed values of the baryon number density $\left(n_{B}=0.32\right.$ and $\left.0.64 \mathrm{fm}^{-3}\right)$ and the same values of $e B, V / S$, and $\mu_{v_{e}}$ used in the previous figures. 
$\alpha_{\text {tot }}^{\perp}$ and $\alpha_{\text {tot }}^{\|}$at PNS conditions are smaller than for the NS case, and for the MERGER case they are even smaller.

In Fig. 4, we show the longitudinal surface tension for electrons. The transverse one is negligible because the contribution of the lowest Landau level to $\alpha_{\text {tot }}^{\perp}$ is zero [see Eq. (20)] and at the same time chemical equilibrium leads in general to low values of the electron chemical potential. The role of finite volume is significant as well as neutrino trapping and temperature effects. The surface tension decreases with the volume, but increases with temperature, neutrinos, and magnetic field.

In Fig. 5 we show the total surface tension in the $\perp$ and $\|$ directions. The surface tension of quark matter in chemical equilibrium under weak interactions is largely dominated by strange quarks (notice that the results in Fig. 5 are very similar to the ones in Fig. 3). The surface tension in the longitudinal and transverse directions is of the order of few $\mathrm{MeV} / \mathrm{fm}^{2}$ at baryon number densities around the nuclear saturation density $n_{0}$ and reaches values of $\approx 15-25 \mathrm{MeV} / \mathrm{fm}^{2}$ around ten times $n_{0}$. The contribution of quarks $u$ and $d$ is always small, with values no higher than $3 \mathrm{MeV} / \mathrm{fm}^{2}$ for the range of baryon number densities of interest (see Figs. 1 and 2). The contribution of electrons is even smaller and negligible in quark matter, with values below $\approx 0.2 \mathrm{MeV} / \mathrm{fm}^{2}$ (see Fig. 4). In summary, we find that for all the astrophysical scenarios considered here the role of different flavors is qualitatively the same as already found in Ref. [1] for cold deleptonized matter.

In Fig. 6, we go beyond the three specific astrophysical cases analyzed before and explore the dependence of $\alpha_{\text {tot }}^{\|}$and $\alpha_{\text {tot }}^{\perp}$ with respect to the temperature for two representative values of the baryon number density, $n_{B}=0.32$ and $0.64 \mathrm{fm}^{-3}$, keeping the same values of $e B, V / S$, and $\mu_{v_{e}}$ as before. In the range of temperatures below $\approx 100 \mathrm{MeV}$, we find that $\alpha_{\text {tot }}^{\|}$ decreases with $T$ while the curves for $\alpha_{\text {tot }}^{\perp}$ are flatter. Above $T \sim 100 \mathrm{MeV}$, the behavior changes and both $\alpha_{\text {tot }}^{\|}$and $\alpha_{\text {tot }}^{\perp}$ grow significantly with $T$.

\section{SUMMARY AND CONCLUSIONS}

In the present work, we have studied the surface tension of hot magnetized quark matter droplets within the formalism of multiple reflection expansion (MRE). Quark matter is described as a mixture of free Fermi gases composed by quarks $u, d, s$ and electrons in chemical equilibrium under weak interactions. We have considered neutrinos in our system in chemical equilibrium with other particles. Even if they do not contribute explicitly to the surface tension (we considered them to be massless), they do affect the surface tension values through the chemical equilibrium condition. Since our system is subjected to the influence of strong magnetic fields, the transverse motion of charged particles is quantized into Landau levels, and as a consequence, the surface tension has a different value in the parallel and transverse directions with respect to $B$. We have considered two values of the magnetic field $\left(e B_{\text {low }}=5 \times 10^{-3} \mathrm{GeV}^{2}\right.$ and $\left.e B_{\text {high }}=5 \times 10^{-2} \mathrm{GeV}^{2}\right)$ and quark matter drops with sizes given by $V / S=10 \mathrm{fm}$, $100 \mathrm{fm}$, and $\infty$. We have considered three different astrophysical scenarios depending on the temperature and neutrino chemical potential: cold deleptonized NSs with $T=1 \mathrm{MeV}$ and $\mu_{v_{e}}=0$, hot lepton-rich PNSs with $T=30 \mathrm{MeV}$ and $\mu_{v_{e}}=100 \mathrm{MeV}$, and binary NS mergers with $T=100 \mathrm{MeV}$ and $\mu_{v_{e}}=200 \mathrm{MeV}$.

For all these astrophysical scenarios, we find that the dependence of the surface tension with the baryon number density, the magnetic field, and the drop's size is essentially the same as found in our previous work at zero temperature and vanishing chemical potential [1]. In fact, we find that for $n_{B}$ between 1 to 10 times the nuclear saturation density, the surface tension falls in the range of $\approx 10^{-2}$ to $25 \mathrm{MeV} / \mathrm{fm}^{-2}$, with the larger contribution coming from strange quarks (the most massive particles in the system). ${ }^{1}$ We also find that the surface tensions in the transverse and parallel directions are almost unaffected by the magnetic field if $e B<e B_{\text {low }}$, but for higher values of $e B$ there is a significant increase in $\alpha_{f}^{\|}$and a significant decrease in $\alpha_{f}^{\perp}$ with respect to the unmagnetized case. Finally, the total surface tension is quite insensitive to the size of the drop, although $V / S$ has a significant effect on the surface tension of light particles (e.g., electrons).

The main differences with respect to previous results are related to the role of trapped neutrinos and finite temperature. We found that at fixed baryon number density, magnetic field, $V / S$, and temperature, both $\alpha_{f}^{\|}$and $\alpha_{f}^{\perp}$ are decreasing functions of the neutrino chemical potential $\mu_{v_{e}}$. The reduction is less than a few percent, but may be relevant for astrophysics because a smaller surface tension makes easier the nucleation of quark matter in a hadronic star. Thus, the hadron to quark conversion is slightly favored by the large amount of trapped neutrinos in the PNS phase and just after a NS merging. We also found that $\alpha_{f}^{\|}$and $\alpha_{f}^{\perp}$ may be significantly affected by temperature. At fixed baryon number density and fixed neutrino chemical potential, we observe that $\alpha_{f}^{\|}$decreases with $T$ in the temperature range of astrophysical relevance ( $T$ below $\approx 100 \mathrm{MeV}$ ), while $\alpha_{f}^{\perp}$ is less sensitive to temperature.

The combined effect of high temperatures and neutrino trapping can be easily assessed from Fig. 5, where we find that, at fixed baryon number density, the surface tension in the

\footnotetext{
${ }^{1}$ The present analysis is based on the standard Massachusetts Institute of Technology (MIT) bag model, which does not include any mechanism for the spontaneous breaking of chiral symmetry. The quark masses are fixed to their current values and do not have any density dependence. More sophisticated models such as the NambuJona-Lasinio model should be employed to describe chiral symmetry breaking. On the other hand, some phenomenological models for the EOS, such as the one presented in Ref. [25], implement quark mass scaling formulas where the "dressed" quark masses depend explicitly on the baryon number density. Within such models, quark masses grow to a few hundred $\mathrm{MeV}$ at some fixed but not too high density. For comparison, we have performed calculations with $m_{u}=m_{d}=$ $300 \mathrm{MeV}$ and $m_{s}=500 \mathrm{MeV}$ in order to test the behavior of the surface tension for typical "constituent" masses. Our results show that the surface tension increases significantly and in some cases it gets close to the critical value $\approx 60 \mathrm{MeV} / \mathrm{fm}^{2}$ above which one expects strong suppression of the mixed hadron-quark phase. A more detailed analysis of the influence of the medium in the quark masses within the MIT bag model is left for future work.
} 
merger case and in the PNS case are always smaller than for cold deleptonized NSs. In the PNS case, these results suggest that quark matter nucleation would be more prone to occur in the earlier evolutionary stages of a hadronic star than in later stages. However, as the star cools and deleptonizes, its radius shrinks and the baryon number density in some layers may increase significantly (typically by a factor of $\approx 2$ [27]). Contrary to the effect of $T$ and $\mu_{v_{e}}$, this may facilitate quark matter nucleation at late stages. Moreover, according to the standard scenario of magnetic field generation in compact stars, the magnetic field inside a PNS could increase during its evolution due to a dynamo mechanism and affect quark matter nucleation if ultrahigh magnetic fields are built inside the object. In the merger scenario, high temperatures, neutrino trapping, and strong magnetic fields are expected to be essential ingredients in the evolution of a hypothetic newly born neutron star [28], but the present theoretical knowledge of the evolutionary stages after coalescence does not allow us to foresee the role of quark matter nucleation in this case.
[1] G. Lugones and A. G. Grunfeld, Phys. Rev. C 95, 015804 (2017).

[2] G. Lugones, A. G. Grunfeld, and M. A. Ajmi, Phys. Rev. C 88, 045803 (2013).

[3] N. K. Glendenning, Compact Stars: Nuclear Physics, Particle Physics, and General Relativity, 2nd ed. (Springer, New York, 2000).

[4] D. N. Voskresensky, M. Yasuhira, and T. Tatsumi, Nucl. Phys. A 723, 291 (2003).

[5] T. Maruyama, S. Chiba, H.-J. Schulze, and T. Tatsumi, Phys. Rev. D 76, 123015 (2007).

[6] C. J. Xia, G. X. Peng, E. G. Zhao, and S. G. Zhou, Phys. Rev. D 93, 085025 (2016).

[7] P. Jaikumar, S. Reddy, and A. W. Steiner, Phys. Rev. Lett. 96, 041101 (2006).

[8] M. G. Alford, K. Rajagopal, S. Reddy, and A. W. Steiner, Phys. Rev. D 73, 114016 (2006).

[9] M. S. Berger and R. L. Jaffe, Phys. Rev. C 35, 213 (1987).

[10] M. B. Pinto, V. Koch, and J. Randrup, Phys. Rev. C 86, 025203 (2012).

[11] T. A. S. do Carmo, G. Lugones, and A. G. Grunfeld, J. Phys. G: Nucl. Part. Phys. 40, 035201 (2013).

[12] E. S. Fraga, M. Hippert, and A. Schmitt, Phys. Rev. D 99, 014046 (2019).

[13] K. A. Bugaev and G. M. Zinovjev, Nuclear Physics A 848, 443 (2010).
[14] G. Lugones and A. G. Grunfeld, Phys. Rev. D 84, 085003 (2011).

[15] A. G. Grunfeld and G. Lugones, Eur. Phys. J. C 78, 640 (2018).

[16] R. Balian and C. Bloch, Ann. Phys. 60, 401 (1970).

[17] J. Madsen, Phys. Rev. D 50, 3328 (1994).

[18] O. Kiriyama and A. Hosaka, Phys. Rev. D 67, 085010 (2003).

[19] O. Kiriyama, Phys. Rev. D 72, 054009 (2005).

[20] M. Strickland, V. Dexheimer, and D. P. Menezes, Phys. Rev. D 86, 125032 (2012).

[21] B. P. Abbott, R. Abbott, T. D. Abbott, F. Acernese, K. Ackley, C. Adams, T. Adams, P. Addesso, R. X. Adhikari, V. B. Adya et al. (LIGO and Virgo Scientific Collaborations), Phys. Rev. Lett. 119, 161101 (2017).

[22] A. Bauswein, T. W. Baumgarte, and H. T. Janka, Phys. Rev. Lett. 111, 131101 (2013).

[23] W. Kastaun and F. Galeazzi, Phys. Rev. D 91, 064027 (2015).

[24] D. Radice, Astrophys. J. Lett. 838, L2 (2017).

[25] C.-J. Xia, G.-X. Peng, T.-T. Sun, W.-L. Guo, D.-H. Lu, and P. Jaikumar, Phys. Rev. D 98, 034031 (2018).

[26] M. Oertel and M. Urban, Phys. Rev. D 77, 074015 (2008).

[27] J. A. Pons, S. Reddy, M. Prakash, J. M. Lattimer, and J. A. Miralles, Astrophys. J. 513, 780 (1999).

[28] B. Giacomazzo, J. Zrake, P. C. Duffell, A. I. MacFadyen, and R. Perna, Astrophys. J. 809, 39 (2015). 